\title{
A Practical Novel Method for Ensuring Stable Capacitation of Spermatozoa from Cryopreserved C57BL/6J Sperm Suspension
}

\author{
Kyuichi TAGUMA $^{1,2,4)}$, Chika NAKAMURA ${ }^{1)}$, Ai OZAKI ${ }^{1)}$, Chigusa SUZUKI ${ }^{1)}$, \\ Akiko HACHISU ${ }^{1,2)}$, Kimio KOBAYASHI ${ }^{1)}$, Keiji MOCHIDA ${ }^{3)}$, \\ Atsuo OGURA ${ }^{3)}$, Hideki KANEDA ${ }^{1)}$, and Shigeharu WAKANA ${ }^{1)}$
}

\footnotetext{
${ }^{1)}$ Technology and Development Team for Mouse Phenotype Analysis, RIKEN BioResource Center, 3-1-1 Koyadai, Tsukuba, Ibaraki 305-0074, 2) JAC Inc., 1-2-7 Higashiyama, Meguro-ku, Tokyo 153-0043, ${ }^{3)}$ Bioresource Engineering Division, RIKEN BioResource Center, 3-1-1 Koyadai, Tsukuba, Ibaraki 305-0074, and ${ }^{4)}$ Present address: Experimental Animal Division, RIKEN BioResource Center, 3-1-1 Koyadai, Tsukuba, Ibaraki 305-0074, Japan
}

\begin{abstract}
A large number of genetically modified mouse strains have been produced in recent years. Sperm cryopreservation is the most effective means of preserving these valuable strains, most of which have a C57BL/6 genetic background. However, the fertilization efficiency of sperm from several cryopreserved strains, including C57BL/6, is quite low. While new and improved methods of cryopreservation have been developed, the majority of sperm stocks have already been cryopreserved using traditional methods, such as storage in $18 \%$ raffinose and 3\% skim milk (R18S3). Therefore, new thawing methods for these frozen stocks are needed. We have developed a new thawing method that involves selective collection of motile sperm and a preincubation medium that enhances capacitation. Motile sperm are selected simply by collecting a sample from the center of a dish, and capacitation is induced by the addition of methyl-beta-cyclodextrin, D-penicillamine, sodium citrate, and hypotaurine to modified Tyrode's solution. The fertilization rate of sperm prepared using this method was increased significantly compared to that of sperm thawed using the traditional method (63.9 vs $16.5 \%, P<0.01)$. These results demonstrate that this new in vitro fertilization method is an effective means of reviving C57BL/6 sperm cryopreserved in R18S3.
\end{abstract}

Key words: capacitation, cryopreservation, C57BL/6J, in vitro fertilization, sperm

\section{Introduction}

Cryopreservation of embryos and gametes is widely used to efficiently archive mouse stocks. Sperm archives are particularly valuable, since in vitro fertilization (IVF) using frozen spermatozoa can be used to rapidly gener- ate large numbers of mice. In most laboratories, sperm are cryopreserved in $18 \%$ raffinose and $3 \%$ skim milk (R18S3) [14, 24]. However, the fertilization efficiency of cryopreserved sperm from several strains (e.g., $\mathrm{C} 57 \mathrm{BL} / 6,129, \mathrm{FVB}$, and BALB/c) is low [22]. Ultrastructural observations suggest that cellular injury caused

(Received 18 February 2009 / Accepted 1 April 2009)

Address corresponding: H. Kaneda, Technology and development team for mouse phenotype analysis, RIKEN BioResource Center, 3-1-1 Koyadai, Tsukuba, Ibaraki 305-0074, Japan 
by cryopreservation is a potential cause of low fertilization rates in the C57BL/6J strain [16]. Freeze-thawed $\mathrm{C} 57 \mathrm{BL} / 6 \mathrm{~J}$ sperm are unable to induce an acrosome reaction and penetrate the zona pellucida of the egg. Moreover, mitochondrial damage leads to a loss of motility.

So far, the production of these difficult strains has required assisted reproductive techniques $[3,11,15]$. However, methods have recently been developed to improve the fertilization rate of cryopreserved C57BL/6 sperm. These include the removal of non-motile sperm and cell debris $[4,19,20]$, preincubation of sperm in medium containing methyl-beta-cyclodextrin (MBCD) [23], or use of a cryoprotective medium containing monothioglycerol [17]. However, the sperm collection process for these methods is complicated, and use of a cryopreservation medium cannot be applied to sperm already frozen in R18S3.

Here, we describe a simple new method of motile sperm selection and preincubation to improve capacitation. Eventually, we aim at the establishment of a stable production system from sperm which have been cryopreserved by a traditional method using R18S3.

\section{Materials and Methods}

\section{Animals}

Inbred male and female C57BL/6J, BALB/cA, BALB/ cByJ, C3H/HeJ, FVB/N, 129/Sv-Ter ${ }^{+}$, DBA/2J, and ICR female mice were obtained from CLEA Japan Inc. (Tokyo, Japan). All experiments conformed to the Japanese regulations on animal care and use, which are based on the Guidelines for Animal Experimentation (Japanese Association for Laboratory Animal Science, JALAS, 1987).

\section{Media}

The basic medium used for sperm preincubation in this study was composed of $0.5 \mathrm{mM}$ MBCD, $0.3 \mathrm{mg} / \mathrm{ml}$ D-penicillamine (penicillamine), $0.5 \mathrm{mM}$ tri-sodium citrate dihydrate $\left(\mathrm{C}_{6} \mathrm{H}_{5} \mathrm{Na}_{3} \mathrm{O}_{7} \cdot 2 \mathrm{H}_{2} \mathrm{O}\right.$; sodium citrate), $0.1 \mathrm{mM}$ hypotaurine, and $1 \mathrm{mg} / \mathrm{ml}$ polyvinyl pyrrolidone in modified Tyrode's solution (Table 1). The fertilization medium used was human tubal fluid medium (HTF) with $0.1 \mathrm{mM}$ hypotaurine. M16 medium (M7292; Sigma Chemical Company, St. Louis, MO) was used to culture the fertilized oocytes.
Table 1. Composition of preincubation medium

\begin{tabular}{|c|c|c|}
\hline \multirow{2}{*}{ Component } & \multicolumn{2}{|c|}{ Concentration } \\
\hline & $\mathrm{mg} / 100 \mathrm{ml}$ & $\mathrm{mM}$ \\
\hline $\mathrm{NaCl}$ & 579.9 & 99.23 \\
\hline $\mathrm{KCl}$ & 20.0 & 2.68 \\
\hline $\mathrm{CaCl}_{2} \cdot 2 \mathrm{H}_{2} \mathrm{O}$ & 26.5 & 1.80 \\
\hline $\mathrm{MgCl}_{2} \cdot 6 \mathrm{H}_{2} \mathrm{O}$ & 10.0 & 0.49 \\
\hline $\mathrm{NaH}_{2} \mathrm{PO}_{4} \cdot 2 \mathrm{H}_{2} \mathrm{O}$ & 5.6 & 0.36 \\
\hline $\mathrm{NaHCO}_{3}$ & 210.0 & 25.00 \\
\hline Glucose & 100.2 & 5.56 \\
\hline Sodium pyruvate & 2.8 & 0.25 \\
\hline $60 \%$ Sodium lactate $(\mu 1)$ & $3.6 \mu 1 / \mathrm{ml}$ & - \\
\hline Potassium penicillin $\mathrm{G}$ & 7.5 & - \\
\hline Streptomycin sulfate & 5.0 & - \\
\hline Phenol red $(\mu 1)$ & 20.0 & - \\
\hline $\mathrm{C}_{6} \mathrm{H}_{5} \mathrm{Na}_{3} \mathrm{O}_{7} \cdot 2 \mathrm{H}_{2} \mathrm{O}$ & 14.7 & 0.50 \\
\hline Methyl- $\beta$-cyclodextrin & $0.7 \mathrm{mg} / \mathrm{ml}$ & 0.50 \\
\hline D-Penicillamine & $0.5 \mathrm{mg} / \mathrm{ml}$ & - \\
\hline Hypotaurine & $0.1 \mathrm{mg} / \mathrm{ml}$ & 0.10 \\
\hline Polyvinyl pyrrolidone & $1.0 \mathrm{mg} / \mathrm{ml}$ & - \\
\hline
\end{tabular}

\section{Sperm freezing and thawing}

Twelve- to twenty-week-old male mice were euthanized, and the caudae epididymides were removed, placed into $100 \mu 1$ of R18S3, and cut into five pieces. Sperm were dispersed for $5 \mathrm{~min}$ at room temperature, and the sperm suspension was divided into $11-\mu 1$ aliquots and carefully loaded into $0.25 \mathrm{ml}$ plastic straws [14]. The straws were then sealed at both ends with a heat sealer, placed in liquid nitrogen vapor for $20 \mathrm{~min}$, then immersed and stored in liquid nitrogen. Frozen sperm suspensions were thawed in a water bath at $37^{\circ} \mathrm{C}$ for $15 \mathrm{~min}$ [14].

\section{Egg collection}

Three- to four-week-old female mice were superovulated using intraperitoneal injections of $5 \mathrm{IU}$ pregnant mare's serum gonadotropin (PMSG, Teikokuzoki Co., Tokyo, Japan) followed by 5 IU human chorionic gonadotropin (hCG, Teikokuzoki Co., Tokyo, Japan) 48 h later. The female mice were sacrificed approximately 15-16 $\mathrm{h}$ after the hCG injection and oocyte-cumulus complexes were collected from the oviducts and placed in a 40- $\mu 1$ drops of fertilization medium covered with mineral oil.

\section{Selective sperm collection method}

The thawed sperm suspension was added directly to 
A

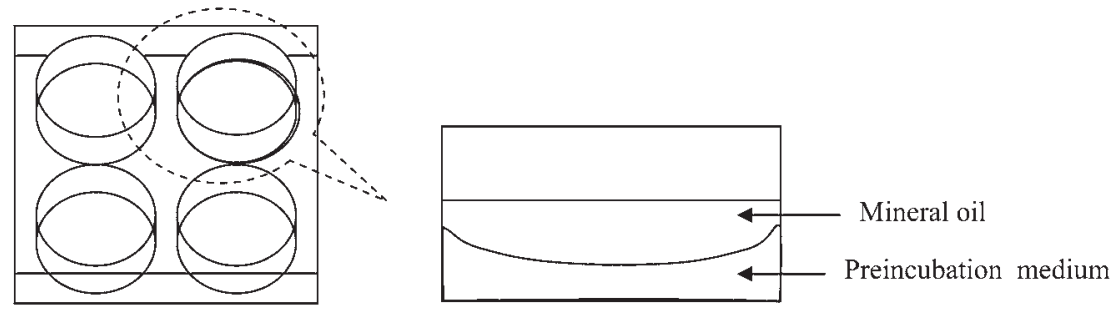

4- well multi dish

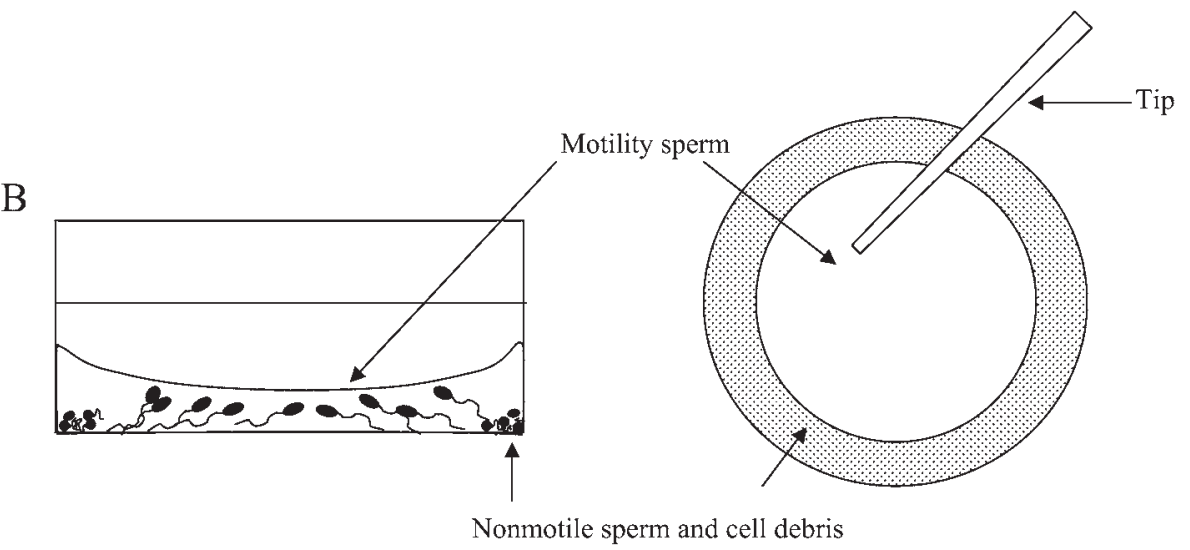

Fig. 1. Selective collection of motile sperm. (A) Sperm are held in preincubation medium in a 4-well dish. (B) Preincubation of sperm resulted in accumulation of nonmotile sperm along the perimeter of the well, concentrating motile sperm at the center of the well.

$220 \mu 1$ of preincubation medium covered with mineral oil in a 4-well dish (No. 176740; Nunc, Roskilde, Denmark). The sperm were preincubated in medium for 60 min at $37^{\circ} \mathrm{C}$ with $5 \% \mathrm{CO}_{2}$ in air. After preincubation, $10 \mu \mathrm{l}$ of motile sperm was collected from the center of the well and added to the two oocyte-cumulus complexes in fertilization medium. After $7 \mathrm{~h}$ in the incubator, oocytes were washed to remove excess sperm and cultured overnight in a 50- $\mu 1$ drop of M16 medium.

\section{Control insemination}

Thawed sperm suspension $(2 \mu 1)$ was added to a 200 $\mu 1$ drop of HTF. After preincubation, two to six oocytecumulus complexes were added to the fertilization medium containing preincubated sperm [14].

\section{Embryo transfer}

Twenty-four hours after insemination, two-cell embryos were counted and transferred into the oviducts of ICR females which had been mated with vasectomized ICR males, on day 1 of pseudopregnancy. After 19 days, the number of pups born was recorded.

\section{Statistical analysis}

Statistical analyses were performed by one-way ANOVA after arcsine transformation of the percentage data using StatView version 5.0 software (SAS Institute Inc.).

\section{Results}

Effect of selective motile sperm collection on fertilization rate

The selective collection method we used is detailed in Fig. 1. Non-motile sperm and cell debris tend to collect around the perimeter of the well, whereas motile sperm tend to accumulate in the center of the well. This phenomenon permits selection of motile sperm simply by collecting from the center of the well. Table 2 shows the effect of selective motile sperm collection on the fertilization rate. The fertilization rates $24 \mathrm{~h}$ after insemination for non-selected (control) and selected sperm were 16.5 and $23.7 \%$, respectively. Thus, the fertility rate of selected sperm was significantly higher $(P<0.01)$ than that of control sperm. 
Table 2. Comparison of in vitro fertilization rates using selected sperm and control sperm from C57BL/6J mice

\begin{tabular}{lccc}
\hline Method & No. of inseminated eggs & No. of 2-cell embryos & Fertilization rate (\%) \\
\hline Control & 401 & 66 & 16.5 \\
Selective collection & 354 & 84 & $23.7^{* *}$ \\
\hline
\end{tabular}

Each experiment was repeated three times. **Values are significantly different from control $(P<0.01)$.

Table 3. Effect of various additives to preincubation and fertilization media on fertility of C57BL/6J mice

\begin{tabular}{|c|c|c|c|c|c|c|c|c|}
\hline \multirow{3}{*}{ Experiment } & \multicolumn{5}{|c|}{ Addition reagent } & \multirow{3}{*}{$\begin{array}{c}\text { No. of } \\
\text { inseminated } \\
\text { eggs }\end{array}$} & \multirow{3}{*}{$\begin{array}{c}\text { No. of } \\
\text { 2-cell } \\
\text { embryos }\end{array}$} & \multirow{3}{*}{$\begin{array}{c}\text { Fertilization } \\
\text { rate } \\
(\%)\end{array}$} \\
\hline & \multicolumn{4}{|c|}{ Preincubation } & \multirow{2}{*}{$\begin{array}{l}\text { Fertilization } \\
\text { Hypotaurine }\end{array}$} & & & \\
\hline & $\begin{array}{l}\text { Methyle- } \beta \text { - } \\
\text { cyclodextrin }\end{array}$ & $\begin{array}{c}\text { D- } \\
\text { Penicillamine }\end{array}$ & $\begin{array}{l}\text { tri-Sodium Citrate } \\
\text { e Dihydrate }\end{array}$ & Hypotaurine & & & & \\
\hline $\mathrm{A}$ & + & + & + & + & + & 1,198 & 766 & 63.9 \\
\hline B & - & + & + & + & + & 236 & 15 & $6.4 * *$ \\
\hline $\mathrm{C}$ & + & - & + & + & + & 193 & 101 & $52.3 * *$ \\
\hline $\mathrm{D}$ & + & + & - & + & + & 325 & 167 & $51.4 * *$ \\
\hline $\mathrm{E}$ & + & + & + & - & - & 500 & 267 & $53.4 * *$ \\
\hline $\mathrm{F}$ & + & + & - & - & - & 168 & 81 & $48.2 * *$ \\
\hline $\mathrm{G}$ & + & - & + & - & - & 291 & 75 & $25.8 * *$ \\
\hline $\mathrm{H}$ & + & - & - & + & + & 241 & 99 & $41.1 * *$ \\
\hline I & + & - & - & - & - & 260 & 79 & $30.4 * *$ \\
\hline $\mathrm{J}$ & - & + & - & - & - & 256 & 9 & $3.5 * *$ \\
\hline
\end{tabular}

Each experiment was repeated three or more times. **Values are significantly different from experiment $\mathrm{A}(P<0.01)$.

\section{Effect of chemical reagents on fertilization rate}

We also tested how the addition of various reagents to the preincubation and fertilization media affected the fertilization rate of frozen C57BL/6J sperm in vitro (Table 3). The fertility of freeze-thawed sperm was enhanced by the addition of MBCD and penicillamine to the preincubation medium and hypotaurine to the fertilization medium. It is likely that this effect is mediated by induction of capacitation. When MBCD, penicillamine, sodium citrate, and hypotaurine were all added to the preincubation medium and hypotaurine to the fertilization medium, the fertilization rate (63.9\%) was significantly higher $(P<0.01)$ than when any individual reagent was added. Thus, these reagents synergistically promote sperm motility, viability, and fertility.

\section{Effect of new in vitro fertilization method on birth rate}

Embryos derived from frozen C57BL/6J sperm thawed as described above were transplanted, and live-born pups were delivered (Table 4). In total, 240 two-cell embryos were transferred to pseudopregnant recipient mice. All of the recipient mice became pregnant, with an aver- age of 11.2 pups born to each. There was no significant difference in developmental capacity between two-cell embryos derived from fresh and frozen sperm.

\section{Effectiveness of new in vitro fertilization method for} other mouse strains

We also examined whether or not our new sperm preparation method improves the fertilization rate in six inbred strains (BALB/cA, BALB/cByJ, FVB/N, C3H/ HeJ, 129/Sv-Ter ${ }^{+}$, and DBA/2J) (Fig. 2). We observed a significant improvement in fertility for the BALB/cBy $(69.3$ vs $50.5 \%, P<0.01)$ and $\mathrm{C} 3 \mathrm{H} / \mathrm{HeJ}(82.5$ vs $38.0 \%$, $P<0.01)$ strains compared with the control method using frozen sperm. This was comparable to the improvement we observed with the C57BL/6J strain (63.9 vs $16.5 \%$, $P<0.01)$. The $129 / \mathrm{Sv}_{-} \mathrm{Ter}^{+}$strain showed an increased fertilization rate with the new method compared to the control freezing method, but the rate remained low. The BALB/cA strain did not show an improvement with the new method, and strains that already showed high fertilization rates with control frozen sperm (FVB/N and DBA/2J) did not show a further increase in the fertiliza- 
Table 4. Post-implantation development of in vitro fertilized embryos from frozen and fresh sperm

\begin{tabular}{lcccc}
\hline Sperm & $\begin{array}{c}\text { No. of } \\
\text { recipients }\end{array}$ & $\begin{array}{c}\text { No. of embryos } \\
\text { transferred }\end{array}$ & $\begin{array}{c}\text { No. of recipients } \\
\text { producing litters }(\%)\end{array}$ & $\begin{array}{c}\text { No. of } \\
\text { offspring (\%) }\end{array}$ \\
\hline Fresh & 10 & 240 & $10(100)$ & $133(55.4)$ \\
Frozen & 10 & 240 & $10(100)$ & $112(46.7)$ \\
\hline
\end{tabular}

The rate of live offspring produced by frozen and fresh sperm did not differ significantly.

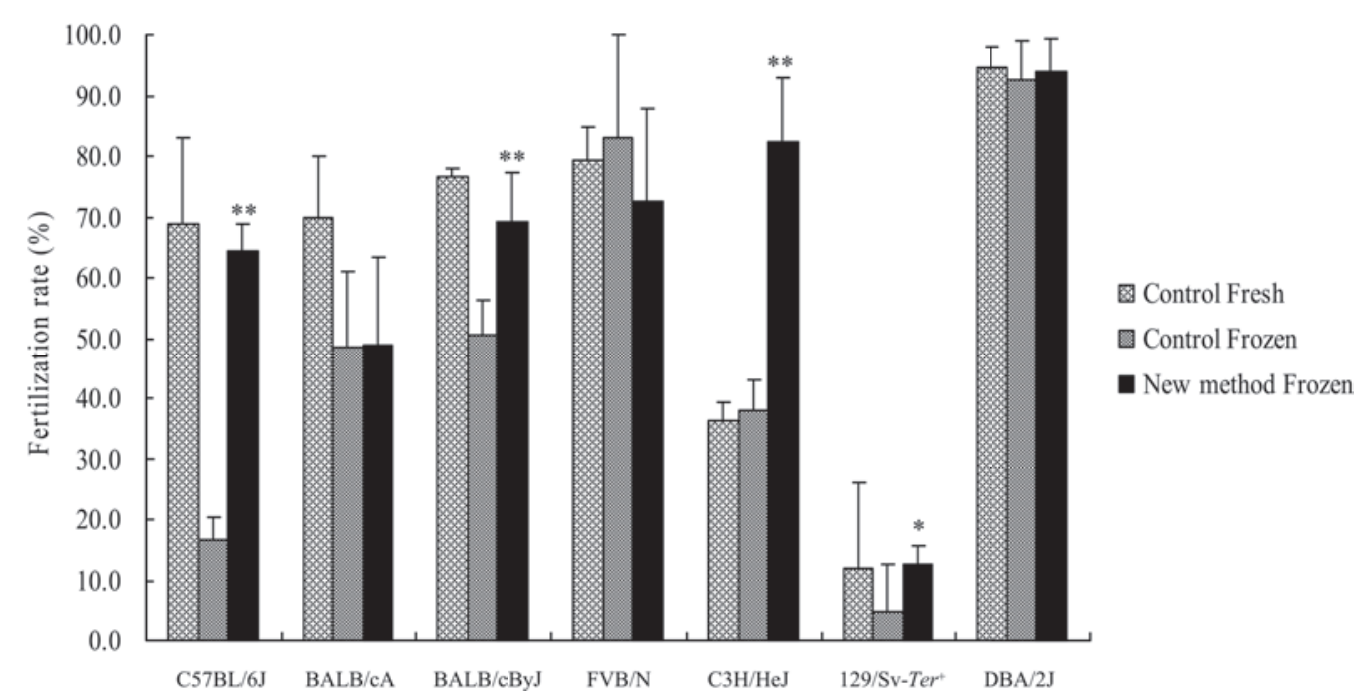

Fig. 2. In vitro fertilization rates in various inbred strains using the new preparation method. The fertilization rate of sperm prepared using our new method (Frozen) was compared to that of sperm prepared with control methods (Frozen and Fresh). Differences between the new and control methods were assessed using ANOVA. The value is the mean \pm SD. *Significantly different from either control method $(P<0.05)$. **Significantly different from either control method $(P<0.01)$.

tion rate with the new method.

\section{Discussion}

We have developed a new method of preparing frozen sperm for in vitro fertilization by selectively collecting motile sperm and preincubating them in a medium to promote capacitation. Previous studies showed that removal of non-motile sperm and cell debris at the prefreeze or postthaw stage increases the fertilization rate of C57BL/6J mouse spermatozoa $[4,20]$. A new method enabling the collection of only motile mouse spermatozoa was developed based on these findings [19], but it involved complicated techniques. In comparison, our method is simple and does not require special equipment. The accumulation of motile sperm at the center of the media may be due to interfacial tension at the dish wall and the collection of these motile sperm resulted in an increased fertilization rates, as previously reported [4, 20].

Previous work has shown that the percentage of damaged C57BL/6J spermatozoa following freezing is $84 \%$, while less than $10 \%$ of frozen $\mathrm{DBA} / 2 \mathrm{~N}$ spermatozoa are damaged [16]. Cryopreservation-induced cellular injury has been suggested as a potential cause of reduced fertilization [16]. Frozen C57BL/6J sperm lose their motility, which is essential for fertilization, due to mitochondrial damage. They are unable to induce an acrosome reaction and cannot penetrate the zona pellucida of an egg due to acrosome damage [16]. However, their fertilization rate can be increased by IVF with partial zona pellucida dissection, zona drilling, or intra- 
cytoplasmic sperm injection [3, 10, 11, 21], suggesting that the low fertilization rate is due to the inability of the sperm to penetrate the zona pellucida.

Damage to the plasma membrane of the sperm head can also prevent fertilization, probably due to inability of the sperm to induce capacitation and the acrosome reaction. Capacitation permits sperm binding and penetration of the zona pellucida. Various physiological changes accompany sperm capacitation, including membrane cholesterol removal, increased protein tyrosine phosphorylation, destabilization of the plasma membrane, increased intracellular calcium levels, and decreased surface charge [8]. In addition to maintaining sperm motility and survivability, BSA facilitates capacitation by causing similar physiological changes, through mechanisms that are largely unknown $[9,12]$.

To improve the fertilization rate of damaged C57BL/6J frozen sperm, we sought to develop a preincubation medium that induces capacitation more effectively than BSA. MBCD stimulates efflux of cholesterol with high efficiency and induces capacitation of fresh sperm [7]. When MBCD is substituted for BSA in a medium, sperm membrane-associated cholesterol is dramatically reduced, and protein tyrosine phosphorylation and capacitation are induced [25]. MBCD is thought to be more effective at removing cholesterol from the membranes of injured frozen sperm with decreased capacitation function [23]. Casein micelles from skim milk prevent removal of cholesterol from sperm plasma membrane, though sperm motility and viability are maintained during cryopreservation [5]. Therefore, sperm frozen in skim milk may need additional cholesterol removal ability compared to fresh sperm.

In addition, removal of $\mathrm{Zn}^{2+}$ from the sperm cell surface by penicillamine destabilizes the plasma membrane preparing the sperm for completion of capacitation and the acrosome reaction, which is a membrane fusion event [2]. Moreover, in fresh ICR sperm, both MBCD and penicillamine are effective at inducing capacitation ( $\mathrm{Fu}-$ kuda personal communication), probably due to synergistic effects on the sperm plasma membrane. We found similar results with frozen sperm.

In our experience, frozen sperm that are preincubated with MBCD tend to lose motility and to die in a short time. This phenomenon is not observed with fresh sperm preincubated with BSA. We found that all freeze-thawed sperm die within about $4 \mathrm{~h}$ after exposure to MBCD (unpublished data), probably because motility is not maintained. For the golden hamster, Japanese grass vole and rabbit, a medium containing the antioxidant hypotaurine maintains sperm survival and motility and improves the fertilization rate $[1,6,26]$, indicating that protection from reactive oxygen species is important for preserving sperm.

Sodium citrate was also supplemented to the medium we used to maintain sperm motility and survival. The osmoregulatory action of citric acid is known to contribute to sperm function in domestic animals $[13,18]$. Citric acid is thought to promote ATP production in sperm mitochondria, thereby providing a source of kinetic energy for the sperm. The amplitude of sperm tailpiece motion and the speed of the sperm are increased by addition of citric acid (unpublished data).

In this study, we were able to improve fertilization rates of freeze-thawed sperm by inducing capacitation by MBCD and penicillamine and enhancing sperm motility and survival by hypotaurine and citric acid. We also selected motile sperm using a simple collection method. Sperm that were prepared using this method showed normal productivity of offspring. It was recently shown that the fertilization rate in various strains, including C57BL/6J, could be improved by including monothioglycerol in the sperm cryopreservation medium [17]. While this is useful for future applications, it does not address how to improve the fertilization rate of the large stocks of sperm that have already been frozen using older techniques. Our method is effective for improving the fertilization efficiency of traditionally cryopreserved sperm from $\mathrm{C} 57 \mathrm{BL} / 6 \mathrm{~J}$ mice, as well as from other strains of mice.

\section{Acknowledgment(s)}

The authors thanks Prof. Yoshinori Fukuda (Veterinary Medicine and Animal Science, Kitasato University) for development of the medium and the technical staff of the Technology and development team for mouse phenotype analysis of RIKEN BRC for animal care. This study was supported in part by the National BioResource Project of the Ministry of Education, Culture, Sports, Science and Technology of Japan. 


\section{References}

1. Alvarez, J.G. and Storey, B.T. 1983. Taurine, hypotaurine, epinephrine and albumin inhibit lipid peroxidation in rabbit spermatozoa and protect against loss of motility. Biol. Reprod. 29: 548-555.

2. Andrews, J.C. and Bavister, B.D. 1989. Hamster zonae pellucidae cannot induce physiological acrosome reactions in chemically capacitated hamster spermatozoa in the absence of albumin. Biol. Reprod. 41: 117-122.

3. Anzai, M., Nishiwaki, M., Yanagi, M., Nakashima, T., Kaneko, T., Taguchi, Y., Tokoro, M., Shin, S.W., Mitani, T., Kato, H., Matsumoto, K., Nakagata, N., and Iratani, A. 2006. Application of laser-assisted zona drilling to in vitro fertilization of cryopreserved mouse oocytes with spermatozoa from a subfertile transgenic mouse. J. Reprod. Dev. 52: 601-606.

4. Bath, M.L. 2003. Simple and efficient in vitro fertilization with cryopreserved C57BL/6J mouse sperm. Biol. Reprod. 68: 19-23.

5. Bergeron, A., Brindle, Y., Blondin, P., and Manjunath, P. 2007. Milk caseins decrease the binding of the major bovine seminal plasma proteins to sperm and prevent lipid loss from the sperm membrane during sperm storage. Biol. Reprod. 77: $120-126$.

6. Boatman, D.E., Bavister, B.D., and Cruz, E. 1990. Addition of hypotaurine can reactivate immotile golden hamster spermatozoa. J. Androl. 11: 66-72.

7. Choi, Y.H. and Toyoda, Y. 1998. Cyclodextrin removes cholesterol from mouse sperm and induces capacitation in a protein-free medium. Biol. Reprod. 59: 1328-1333.

8. de Lamirande, E., Leclerc, P., and Gagnon, C. 1997. Capacitation as a regulatory event that primes spermatozoa for the acrosome reaction and fertilization. Mol. Hum. Reprod. 3: 175-194.

9. Go, K.J. and Wolf, D.P. 1985. Albumin-mediated changes in sperm sterol content during capacitation. Biol. Reprod. 32: $145-153$.

10. Kawase, Y., Aoki, Y., Kamada, N., Jishage, K., and Suzuki, H. 2004. Comparison of fertility between intracytoplasmic sperm injection and in vitro fertilization with a partial zona pellucida incision by using a piezo-micromanipulator in cryopreserved inbred mouse spermatozoa. Contemp. Top. Lab. Anim. Sci. 43: 21-25.

11. Kawase, Y., Iwata, T., Ueda, O., Kamada, N., Tachibe, T., Aoki, Y., Jishage, K., and Suzuki, H. 2002. Effect of partial incision of the zona pellucida by piezo-micromanipulator for in vitro fertilization using frozen-thawed mouse spermatozoa on the developmental rate of embryos transferred at the 2-cell stage. Biol. Reprod. 66: 381-385.

12. Loeser, C.R. and Tulsiani, D.R. 1999. The role of carbohydrates in the induction of the acrosome reaction in mouse spermatozoa. Biol. Reprod. 60: 94-101.

13. Mesáros, P., Gamcík, P., and Schvarc, F. 1977. Ram sperm survival during the use of various solvents after deep freezing in liquid nitrogen. Vet. Med. (Praha) 22: 599-
604.

14. Nakagata, N. 2000. Cryopreservation of mouse spermatozoa. Mamm. Genome. 11: 572-576.

15. Nakagata, N., Okamoto, M., Ueda, O., and Suzuki, H. 1997. Positive effect of partial zona-pellucida dissection on the in vitro fertilizing capacity of cryopreserved C57BL/6J transgenic mouse spermatozoa of low motility. Biol. Reprod. 57: 1050-1055.

16. Nishizono, H., Shioda, M., Takeo, T., Irie, T., and Nakagata, N. 2004. Decrease of fertilizing ability of mouse spermatozoa after freezing and thawing is related to cellular injury. Biol. Reprod. 71: 973-978.

17. Ostermeier, G.C., Wiles, M.V., Farley, J.S., and Taft, R.A. 2008. Conserving, distributing and managing genetically modified mouse lines by sperm cryopreservation. PLOS ONE 3: e2792.

18. Salamon, S. and Ritar, A.J. 1982. Deep freezing of Angora goat semen: effects of diluent composition and method and rate of dilution on survival of spermatozoa. Aust. J. Biol. Sci. 35: 295-303.

19. Suh, R., Takayama, S., and Smith, G.D. 2005. Microfluidic applications for andrology. J. Androl. 26: 664-670.

20. Szczygiel, M.A., Kusakabe, H., Yanagimachi, R., and Whittingham, D.G. 2002. Separation of motile populations of spermatozoa prior to freezing is beneficial for subsequent fertilization in vitro: a study with various mouse strains. Biol. Reprod. 67: 287-292.

21. Szczygiel, M.A., Kusakabe, H., Yanagimachi, R., and Whittingham, D.G. 2002. Intracytoplasmic sperm injection is more efficient than in vitro fertilization for generating mouse embryos from cryopreserved spermatozoa. Biol. Reprod. 67: 1278-1284.

22. Sztein, J.M., Farley, J.S., and Mobraaten, L.E. 2000. In vitro fertilization with cryopreserved inbred mouse sperm. Biol. Reprod. 63: 1774-1780.

23. Takeo, T., Hoshii, T., Kondo, Y., Toyodome, H., Arima, H., Yamamura, K., Irie, T., and Nakagata, N. 2008. Methyl-betacyclodextrin improves fertilizing ability of C57BL/6 mouse sperm after freezing and thawing by facilitating cholesterol efflux from the cells. Biol. Reprod. 78: 546-551.

24. Takeshima, T., Nakagata, N., and Ogawa, S. 1991. Cryopreservation of mouse spermatozoa. Exp. Anim. 40: 493-497.

25. Visconti, P.E., Galantino-Homer, H., Ning, X., Moore, G.D., Valenzuela, J.P., Jorgez, C.J., Alvarez, J.G., and Kopf, G.S. 1999. Cholesterol efflux-mediated signal transduction in mammalian sperm. beta-cyclodextrins initiate transmembrane signaling leading to an increase in protein tyrosine phosphorylation and capacitation. J. Biol. Chem. 274: 3235-3242.

26. Wakayama, T., Suto, J., Imamura, K., Toyoda, Y., Kurohmaru, M., and Hayashi, Y. 1996. Effect of hypotaurine on in vitro fertilization and production of term offspring from in vitrofertilized ova of the Japanese field vole, Microtus montebelli. Biol. Reprod. 54: 625-630. 